\title{
PSICOPATOLOGÍA Y “TEORÍA DE LAS RELACIONES OBJETALES”
}

\section{PSYCHOPATHOLOGY AND “OBJECT RELATIONS THEORY”}

\section{Manuel Martínez Herrera*}

\author{
RESUMEN
}

El presente artículo ubica los principales cuadros psicopatológicos a través de lo propuesto por la Teoría de las Relaciones Objetales, siguiendo a tal efecto una visión longitudinal del individuo desde una perspectiva clínica-dinámica. Se abordan en este recorrido, las denominadas funciones maternas y paternas, así como, los principales dilemas y tareas evolutivas de cara a la construcción de la subjetividad.

PALABRAS CLAVE: PSICOLOGÍA * TEORÍA DE LAS RELACIONES OBJETALES * PSICOPATOLOGÍA * SUBJETIVIDAD * MADRE

\section{ABSTRACT}

This article analyzes the main psychopathological disorders related to the exposed by the Object Relations Theory, considering therefore a lengthwise vision of the individual from a clinical-dynamic perspective. It is exposed the maternal and paternal functions, the main dilemmas and evolutionary tasks facing the construction of subjectivism.

KEYWORDS: PSYCHOLOGY * OBJETAL RELATIONS THEORY * PSYCHOPATHOLOGY * SUBJECTIVITY * MOTHER 


\section{INTRODUCCIÓN}

Históricamente, el Psicoanálisis ha derivado en múltiples desarrollos teóricos desde que Freud lo formulara oficialmente, en 1900, en su obra inaugural La Interpretación de los Sueños, la cual consideraba su obra más importante. Desde entonces, la obra freudiana ha sido sometida a múltiples reformulaciones que se debaten entre sí, con marcadas e importantes diferencias a nivel epistémico, óntico y heurístico, que en todo caso, más allá de las discrepancias, han contribuido a la construcción de teorías y métodos en diversos campos, tales como: el ámbito clínico, la lingüística, la hermenéutica y el análisis social, entre otros.

Recapitulando la evolución histórica del psicoanálisis, Braunstein (1993) establece la existencia de cuatro lecturas psicoanalíticas, de las cuales una de ellas es debido a la autoría de Anna Freud, hija del propio Sigmund Freud. La autora enfatiza en las distintas tópicas freudianas, particularmente, en la última de ellas, en la cual su padre establece la existencia de las instancias psíquicas del ello, el yo y el súper yo, así como del "comercio psíquico" entre ellas, según la expresión del mismo Freud, para referirse a su concepción dinámica (Brenner, 1988). Por su parte, Anna brinda una extraordinaria importancia a los distintos mecanismos de defensa al servicio del funcionamiento $y$ adaptación del yo a la realidad (Freud, A., 1954), relevancia que no se encuentra en Freud, ni siquiera en sus historiales clínicos. Históricamente, dicha lectura se convirtió en el psicoanálisis oficial avalado por el propio Freud y aprobado por la Asociación Psicoanalitica Internacional (API) (en inglés, International Psychoanalytical Association- IPA).

Por otra parte, el Freud de Melanie Klein (1948), inspirado en las concepciones biologistas del Freud (1915) médico y neurólogo, quien prometió que algún día la ciencia —obviamente, se refería a las ciencias médicas - daría cuenta de sus hallazgos, particularmente, de lo que denominó el "quimismo de la sexualidad". Es el Freud instintivista $y$ pulsional, del edipo $y$ el súper yo temprano de la denominada Escuela Inglesa. Ante los desarrollos del psicoanálisis annafreudiano y kleiniano, el viejo Freud (1932) toma partido, sin lugar a dudas, por la propuesta de su hija, que era de por sí más afín a su propio pensamiento.

Existe también el Freud de la palabra y de la interpretación, este es el Freud (1905, 1909 y 1911) de los historiales clínicos y en otro sentido, existe el Freud con la posibilidad de acceder al contenido inconsciente, a partir de la re-simbolización y la resignificación, el cual será el Freud de Lacan.

Por último, está el Freud de los textos culturales (1912, 1921, 1927 y 1930), el de la relación y tensión entre el sujeto y la cultura, de la psicogénesis $y$ la sociogénesis en tanto $y$ en cuanto, dimensiones del ser (Martínez, 2013). Es el Freud de la Escuela Crítica y de los denominados culturalistas.

Realizada esta ubicación necesariamente somera de los principales desarrollos históricos del Psicoanálisis, es preciso indicar que en este texto se referirá a la tradición kleiniana, la cual en criterio del autor es la que realiza la mayor aportación a la psicología evolutiva desde una perspectiva analítica y constituye uno de los grandes discursos de la psicopatología clínica contemporánea. Particularmente, la Teoría de las Relaciones Objetales brinda un importante aporte al Psicoanálisis en la comprensión de la constitución de la subjetividad, las relaciones vinculares, los vínculos afectivos y los complejos y psicopatologías resultantes, de particular interés para la presente propuesta. Bajo el concierto de la Teoría de las Relaciones Objetales, inspirada por la propia Melanie Klein (1948), se abordará la génesis y el desarrollo de la psicopatología desde una perspectiva constitucional y estructural.

\section{DESARROLLO}

El inicio de la vida de todo ser humano evoca el caos, el cual se asume que caracterizó el surgimiento de la materia, los planetas y posteriormente, de la vida en los albores de los tiempos. La criatura humana en el momento mismo de su nacimiento $y$ durante algún tiempo, se encuentra en un periodo pre-lingüístico y carente de toda estructura, donde no discierne el "yo" del "no yo", el "yo" del "otro"; en este periodo no es capaz de concebir la otredad porque 
no puede concebirse así mismo. Este es el estado que Freud, haciendo acopio de las tradiciones orientales denominó "el estado de nirvana", por cuanto se es uno con la totalidad; empero, a diferencia del nirvana oriental, el nirvana freudiano no ocurre a partir de un extraordinario desarrollo y expansión de la conciencia, por el contrario, obedece a un precario y primigenio desarrollo del individuo carente de conciencia $y$ donde no existe una diferenciación con la totalidad. Este momento original del desarrollo, se ha denominado según distintos autores y autoras, la fase ego-cósmica, egocéntrica o autística -en este último caso no se hace referencia al síndrome clínico del autismo- todo lo cual, corresponde al narcisismo primario o narcisismo absoluto freudiano (Freud, 1914), que es un narcisismo sin objeto, es decir, donde no existe la noción del otro, a partir de lo cual me constituyo como sujeto.

$\mathrm{Al}$ advenimiento a la vida, el ser humano se encuentra preso de su concretad. A efecto de tener una idea más o menos aproximada de los fenómenos que acaecen en este periodo y a falta de una huella mnémica o de una inscripción de la ausencia de dicho momento primordial que pudiera evocarlo de alguna manera, se utilizarán algunas metáforas para acceder a la comprensión de estos fenómenos, después de todo, el lenguaje es siempre una metáfora que da cuenta de la realidad, a partir de la palabra que la representa y la simboliza.

Desde el punto de vista visual —una de las principales formas de contacto con el mundo exterior-, los recién nacidos "perciben" el entorno como si lo vieran a través de una poderosa lente desenfocada, esto es, un mundo de formas sin forma, de colores que no son tales, ya que el aprendizaje de estos es una tarea de la educación preescolar y evolutivamente tardía, en relación al momento vital al que se refiere.

Para hablar tan solo de otro sentido sensorial esencial como lo es la audición, se dirá que el mundo sonoro no es menos caótico, ya que es percibido como un conjunto de ruidos sin que se reconozca su procedencia, naturaleza y mucho menos su sentido. Solo un ruido súbito y fuerte es capaz de excitar y estremecer a la pequeña criatura quebrando su precaria homeostasis, lo que provoca el llanto sin poder discernir que dicho ruido es de una naturaleza diferente a un cólico que también le hace llorar. Esto obedece a que, desde el punto de vista endogénico, durante las primeras semanas de vida, los bebés no son capaces de reconocer lo propio y lo ajeno, ni tampoco lo de adentro o lo de afuera. Estas experiencias intra y extracorpóreas no se reconocen como diferentes a partir de su origen y naturaleza.

Por otra parte, el esquema corporal que ha tenido un insipiente desarrollo en el momento evolutivo del que se habla, poco a poco se va integrando al reconocimiento de las primeras partes del cuerpo que se asumen como propias. La mano, que en un movimiento azaroso es llevada por la criatura a su boca, procurándole placer, se troca posteriormente, en un movimiento dirigido intencionadamente a la zona oral, estableciéndose así un esquema; lo propio acontece con los dedos de los pies, que terminará también reconociéndolos como suyos.

Este estado previo a la alteridad, carente de sentido, forma, organización y temporalidad alguna, es lo que Klein denomina el estado autístico. Ahora bien, a partir de este mundo bizarro en que se encuentra sumergido el ser humano, acaece alrededor del segundo o tercer mes de vida, el reconocimiento de un primer objeto de su entorno, que según los experimentos de Spitz (1977), es el rostro humano, al cual el niño y la niña dirigen una sonrisa responsiva a un semejante, a una otredad más allá de sí mismo, de lo cual se colige la vocación onto y filogenética que a nivel social caracteriza al ser humano. No es de extrañar que el primer discernimiento del universo visual sea el rostro humano, habida cuenta de que se ha comprobado experimentalmente, que durante las primeras semanas y meses de vida, los bebés reaccionan a determinados estímulos próximos - cercanos a los veinte centímetros- de su campo visual, que es aproximadamente la distancia que separa los rostros de la madre y el bebé durante el amamantamiento. Tampoco es azaroso que dentro de los primeros "avistamientos" se encuentre el pecho materno asociado a funciones oral-alimenticias vitales $y$ vinculado al apego afectivo, ya que el amamantamiento 
es por antonomasia, el primer vínculo que se establece con un otro significativo, quien gratifica $y$ resarce las necesidades emocionales $y$ de seguridad de la joven criatura.

Al anterior reconocimiento del rostro y del pecho materno o de quien lo sustituya, se suma la voz humana que emerge de esta totalidad indiscriminada en el universo sonoro e indefinido, en la cual se habita. Sin embargo, es una voz incierta e indeterminada, no es la voz de alguien en particular que se le reconoce como tal. Lo anterior, es lo que se denomina en Teoría de las Relaciones Objetales, los "objetos parciales" (Klein, 1948), ya que aún no ha advenido el objeto afectivo por excelencia que es siempre la madre o quien cumpla su función, al respecto se volverá más adelante sobre dicho tema. Una de las características de los objetos parciales es precisamente que no existe la integración de un otro significativo, sino tan solo percepciones de gratificación e insatisfacción asociadas a la presencia o ausencia de tales objetos, que justamente se caracterizan por su indiscriminación, al no ser factible asociarlos y asimilarlos a una persona en particular en este momento vital.

En relación con los objetos parciales, Hinshelwood, Robinson y Zárate (1997) introducen la noción de Klein sobre el "pecho bueno" y el "pecho malo", que precisamente alude a aquel pecho que en presencia resarce en lo oral alimenticio y en lo afectivo, pero que en ausencia no gratifica $y$ frustra. Estas son las dimensiones del placer y el displacer freudiano (1915) que se encuentran como experiencias esenciales en el origen mismo de la existencia. Al igual que el pecho y el rostro, la voz humana en presencia, se asocia prontamente a gratificación y protección, en tanto, su ausencia se asocia a insatisfacción.

Continuando con el desarrollo longitudinal del ser humano, se tiene que alrededor del cuarto mes de existencia (Spitz, 1977), ocurre un reconocimiento de un rostro en particular que destaca de los demás rostros indiferenciados e indiscriminados. De esta manera, la criatura humana es capaz de reconocer un rostro primordial y esencial, lo propio ocurre con el pecho y con la voz, es decir, que entre todas las presencias discierne e integra la presencia materna o de quien asuma dicha representación. Así es como cuando el niño o niña claman por la figura materna, solo su exclusiva presencia es capaz de satisfacer su demanda. Este es un momento crucial en el desarrollo de la subjetividad humana, donde por primera vez se reconoce un otro, un semejante, un igual vinculado pero a la vez diferenciado de sí mismo, en quien se deposita la confianza y la seguridad.

Este hecho trascendental marca un acontecimiento de extraordinaria importancia psicológica, como lo es el establecimiento de un vínculo con un objeto afectivo, el cual será el prototipo y arquetipo de las relaciones vinculares ulteriores que moldeará, como nunca antes y después, los aspectos constitucionales y estructurales que nos definirán como sujetos.

A partir del establecimiento de la relación objetal, se funda $y$ desarrolla un vínculo esencial, cuyas manifestaciones visibles se pueden localizar con mayor certeza a partir del cuarto mes de vida, siendo el objeto afectivo materno sujeto de reconocimiento $y$ demanda incondicional. En este momento de la vida, se encuentra la angustia de separación, tal y como la definió Spitz (1977), la cual no se aplaca con ninguna otra presencia humana que no sea la de la madre o su sucedánea. Es así como el niño o la niña exigen una presencia determinada, que le es entrañable y a la cual reconocen claramente, cuya ausencia provoca una primera angustia que precisamente se denomina "de separación". La "angustia de separación" se constituye en el prototipo de las posteriores ansiedades $y$ neurosis, su existencia revela que los niños y las niñas no han logrado la permanencia psicológica del objeto afectivo, por lo cual requieren de su constante presencia para su satisfacción y resarcimiento afectivo, así como para su seguridad emocional.

Posteriormente, se irá desarrollando lo que se ha denominado constancia objetal o permanencia del objeto afectivo, que no es otra cosa más que la satisfacción inicialmente momentánea que brinda la actividad lúdica simbólica, a partir del recuerdo del objeto; lo propio ocurre con la representación simbólica que da pie a lo que Winnicott (1971) llamó el "objeto 
transicional", todo lo cual brinda una determinada seguridad emocional $y$ una suerte de pacto de esperanza, basado en la experiencia de que el objeto afectivo siempre retornará. El propio Freud describió magistralmente este proceso, a partir del juego de una de sus hijas con un carrete de hilo, que la niña alejaba y acercaba de sí misma simbolizando la ausencia y presencia de la madre.

Este vínculo particularmente estrecho $y$ profundo entre la madre $y$ su hijo o hija, se denomina simbiosis (Mahler, 1972). La simbiosis es un concepto de la Biología que la Psicología asume para sí, el cual describe una interdependencia entre dos organismos, en nuestro caso, entre la madre y el hijo o la hija. Dicha interdependencia es clara y evidente en el caso de la pequeña criatura humana, ya que ese habla del quinto o sexto mes de vida y evidentemente, este no tiene ninguna oportunidad de sobrevivir sin los cuidados y auxilios de un adulto. Igualmente, la madre o quien la representa, genera una relación simbiótica con el niño o la niña, al punto de establecer una fusión dual, de manera tal que madre e hijo o hija terminan siendo uno. Las madres que han tenido la trágica experiencia de perder a un hijo o hija alrededor de los seis meses de edad, indefectiblemente dicen en lo literal y en lo simbólico lo mismo: "es que me arrancaron un pedazo de mí". Dicha sensación tiene su explicación en la simbiosis, ya que la madre o quien cumpla su función se encuentra fusionada en uno con el hijo o la hija $y$ su pérdida se vive como una especie de desmembramiento. El "sueño de nodriza" descrito por Freud — según el cual casi ningún ruido, por fuerte que sea, perturba su sueño-, implica que la madre se despierta inmediatamente ante el mínimo susurro del bebé a su cargo, lo cual es otro claro ejemplo de relación simbiótica. Por su parte, el llamado que hacen las pequeñas criaturas con sus balbuceos convocando a la madre, son también parte del proceso simbiótico como tal, incluso, hay quienes cifran el origen del lenguaje a partir de la necesidad de invocar a la madre.

En el periodo de la simbiosis, la madre o quien realiza su función, es quien califica la experiencia del niño o la niña, es decir, si la experiencia relacional con la madre es fundamentalmente buena, el niño o la niña se van a sentir buenos $y$ amados, si por el contrario, la experiencia con la madre no es buena, el niño o la niña se van a sentir malos y rechazados. En este momento de la vida del individuo, no existe posibilidad alguna de tener un juicio crítico independiente que le exima de responsabilidad frente al designio materno, de allí la extraordinaria importancia de este proceso vincular. Dichas experiencias primarias a nivel objetal, dejarán una marca indeleble, de manera tal que determinarán aspectos constitucionales y estructurales que nos definirán como sujetos, a partir de sus consecuencias positivas y negativas. El re-aseguramiento afectivo que significa, según Erickson (2000), la "confianza básica versus la desconfianza básica”, es un proceso que se encuentra en el fundamento del edificio de la personalidad $y$ acontece durante el primer año de vida, dicho proceso encuentra su núcleo duro en las relaciones vinculares, a partir del objeto afectivo primario.

Desde el punto de vista psico-etiopatogénico, se puede decir que el periodo de la simbiosis es el lugar de origen de los trastornos constitucionales y estructurales, también definidos como trastornos de la personalidad (Asociación Americana de Psiquiatría, 2002), que implican rasgos y modalidades comportamentales persistentes a nivel cognitivo, afectivo, interpersonal y social. Estas modalidades suelen mantenerse a lo largo de la vida, más allá de las cambiantes situaciones socio-culturales y frecuentemente, provocan dificultades de funcionamiento $y$ de adaptación social.

Por ejemplo, en el llamado trastorno de personalidad dependiente (Asociación Americana de Psiquiatría, 2002), acaece una simbiosis no resuelta que trasciende el momento evolutivo de su superación que frecuentemente, persiste durante toda la infancia, la adolescencia y la vida adulta. Este trastorno es prohijado por una madre o su representante con rasgos de personalidad dependencial, cuando no con un trastorno de personalidad dependiente propiamente dicho, encuentra en el hijo o hija su amparo y un soporte afectivo y emocional, sin el cual, fácilmente se derrumba. Este tipo de madre lanza 
una suerte de consigna más o menos explícita a su hijo o hija que dice aproximadamente así: "Hijo mío, hija mía, el mundo es un lugar peligroso, solo a mi amparo y en mi regazo encontrarás protección". La pequeña criatura aprende a partir de la experiencia compartida con la madre - quién califica la experiencia - a vivir el mundo y la vida como algo amenazante e incluso terrorífico, a no confiar en sí mismo ni en los demás, únicamente en la madre.

Dicha situación es particularmente, frecuente en el caso de las madres ansiógenas, lo cual deriva en la necesidad de una presencia omnipresente de la figura materna para el reaseguramiento emocional y narcisístico del niño y la niña, así como de la madre. Estas madres suelen ser personalmente inseguras $y$ al no confiar en sí mismas, tampoco confían en su "obra" o su "producto", que es su hijo o hija. Suelen ser también personas inhibidas, ansiosas y temerosas, de manera tal, que al estar atrapadas en un proceso vincular de naturaleza simbiótica, transfieren sus propios miedos y ansiedades a sus hijos e hijas (Liberman, 1984).

En estas circunstancias, la progenie no hace otra cosa más que reproducir los miedos $y$ ansiedades de la madre, inculcados y sostenidos por esta. Dicha modalidad relacional se convierte en el arquetipo vincular, llevando a estos individuos más adelante a establecer relaciones, en donde demandan a los otros, cuidado y protección, constituyéndose los demás en sucedáneos maternos, ya sea el padre, el grupo fraterno, la pareja, las amistades, los compañeros y compañeras de trabajo o estudio entre otras muchas posibilidades, incluido por supuesto, los y las psicoterapeutas. Este tipo de relación vincular se constituye en un rasgo caractereológico que determina la vida de los sujetos, en donde el otro funciona como una suerte de alter-ego o yo auxiliar, siendo a la vez fuente indispensable de confianza, seguridad y confirmación del propio yo.

En el trastorno paranoide de la personalidad (Asociación Americana de Psiquiatría, 2002), se encuentra la existencia de un fuerte defraudamiento primario, en términos de que el objeto afectivo primordial no resarce, ni libidinal ni narcisistamente al individuo
(Liberman, 1984), quien de esta manera, sufre una afectación en lo que Ericksson (2000) denominó "confianza básica". Dicho defraudamiento fundamental — que acaece en términos del primer año de vida - repercutirá a lo largo de la existencia, al fin $y$ al cabo, sino se puede confiar en la madre (psicológica), ¿en quién se puede confiar? La confianza cifrada en un otro significativo que no es correspondida, refuerza la posición que Melanie Klein denominó "esquizo-paranoide" (Hinshelwood, Robinson y Zárate, 1997), según la cual no solo se desconfía de los demás, sino que se les teme verbigracia de la proyección de las propias pulsiones de destrucción y muerte, que en virtud de dicho mecanismo, devienen como amenazas del exterior, resultado de un abandono o una agresión real y fantaseada, por lo cual no se espera de los otros, más que daño y traición.

En relación a las constituciones de naturaleza narcisista (Asociación Americana de Psiquiatría, 2002), existe una sobrecarga más allá de lo normal y conveniente de libido materna, en desmedro de las disposiciones necesarias para enfrentar la inevitable condición de la frustración, la cual fortalece el criterio de realidad, ya que ubica los límites y contornos del "yo", en los contextos interpersonales y socioculturales, impidiendo que el ego se expanda narcisísticamente sin control alguno. En dicho trastorno, los sentimientos de omnipotencia tampoco encuentran su límite y se provee al sujeto de un desmesurado criterio acerca de la propia valía e importancia en detrimento de los demás. Para estas madres, los hijos e hijas son posesiones y realizaciones personales, verdaderas extensiones de sí mismas (Liberman, 1984), los cuales tienden a operar al tenor de la siguiente metáfora: "Hijo mío, hija mía, eres lo más grande y maravilloso del mundo y de la vida y lo mereces todo, ve y tómalo".

En el periodo simbiótico, se ha dicho que la madre o quien cumpla su función, es la que califica y determina la experiencia del niño $y$ de la niña, ya que no es posible en dicho periodo de la vida, sustraerse de los dictados maternos. Asimismo, en este periodo, el mandato materno una vez establecido, es indudable e incuestionable, de allí que aquellos sujetos que queden 
atrapados en el universo significativo materno, se asumen como superiores y con prerrogativas especiales, existentes solo en el discurso materno y en el espacio construido por la relación simbólica, única realidad con que se cuenta.

La personalidad antisocial (Méndez, 2003) puede ser explicada a partir de una acumulación libidinal del sujeto sobre sí mismo(a) en detrimento de los objetos afectivos, debido a un defraudamiento primario de naturaleza "maligna", concepto utilizado por Otto Kemberg (1989). Bien decía Freud (1915) que las necesidades pulsionales pueden ser satisfechas de manera autoerótica o requerir de un objeto afectivo externo para su satisfacción. En caso de que dicho objeto afectivo sea omiso, rechazante o destructivo, esas pulsiones retornan narcisísticamente al sujeto que se convierte en el propio objeto afectivo, mostrándose autocomplaciente $y$ tendiendo a satisfacer sus necesidades a expensas de los demás. De allí, la incapacidad constitucional a nivel empático que muestran las estructuras sociopáticas, las cuales solo propenden a la propia satisfacción y que tienen como único objeto afectivo al propio yo.

Se podrían brindar otras explicaciones psicodinámicamente, orientadas en relación a otros trastornos de la personalidad; sin embargo, basta con lo expuesto hasta aquí, para establecer el valor fundante $y$ determinante de la vinculación primaria en la constitución de la subjetividad y su impacto no solo a nivel psicopatológico, sino también en cuanto a las orientaciones básicas de la personalidad.

Desde una perspectiva ontológica, el vínculo primario no solo es fundamental para el aseguramiento emocional, si no que es vital en el sentido lato del término. Los clásicos estudios acerca de la deprivación afectiva de John Bowlby (1991) y Rene Spitz (1977), demuestran los efectos devastadores que provoca una ruptura abrupta del vínculo afectivo entre madre-hijo o hija, cuyas consecuencias en los ámbitos de la salud física, el desarrollo y a nivel emocional pueden provocar en niños y niñas menores de un año, la denominada "depresión anaclítica", el "marasmo" e incluso la muerte, aún en condiciones de una adecuada atención médica. Ambas condiciones se encuentran desde una perspectiva etiológica directamente relacionadas con la pérdida del objeto afectivo.

Tal y como ha quedado establecido, en el proceso de simbiosis, la madre tiene la llave de acceso al mundo $y$ a la vida o al atrapamiento en su universo simbólico. Las madres seguras de sí mismas confían en sus hijos e hijas y les proponen "irrumpir" en la realidad, dicha posibilidad hace que hijos e hijas tengan una creciente autonomía, que como proceso es paulatino y demanda tiempo. Las paternidades y las maternidades que posibilitan experiencias autonómicas - que inevitablemente conllevan episodios de frustración-, brindan la posibilidad de un desarrollo más pleno de las capacidades y potencialidades infantiles, preparándolos de mejor manera para una eficaz inserción social. Es importante señalar en lo que a las experiencias se refiere - gratificantes y frustrantes-, que estas deben propiciar vivencias con una valencia fundamentalmente positiva, de manera tal, que cuando se enfrenten experiencias negativas, el individuo se encuentre reasegurado y fortalecido para superar los obstáculos y manejar la frustración de manera satisfactoria con esperanza y confianza.

La etapa de separación/individuación (Mahler, 1972) que acaece alrededor del primer año de vida, es una oportunidad que brinda la madre o quién la representa para un creciente ejercicio de la libertad y la autonomía. A partir de la separación de la figura materna, quien ya no califica más la experiencia del niño y la niña a diferencia de la simbiosis, estos se individualizan como otro u otra diferenciados de la madre.

Durante la posición esquizo-paranoide de Melanie Klein (Hinshelwood, Robinson y Zárate, 1997), las pulsiones de destrucción y de muerte originalmente dirigidas al objeto afectivo malo - que en ausencia frustra-, devienen como amenazas externas contra el propio sujeto verbigracia de un mecanismo de proyección primitivo, amenazándolo con la aniquilación, de allí la naturaleza persecutoria y paranoica de dicha posición. Sin embargo, a partir de la superación de la posición esquizo-paronoide $y$ de la simbiosis, la proyección de las pulsiones de destrucción y de muerte ya no son posibles, por lo cual el individuo debe hacerse cargo de las 
propias pulsiones, sean estas buenas o malas, integrándolas como parte de sí.

Siempre y cuando la madre o quien la representa abra la puerta $y$ permitan al hijo o la hija acceder al mundo y a la vida, se arribará a la etapa denominada separación e individuación, que consiste precisamente en el rompimiento de la simbiosis a partir de lo cual, la criatura humana se sabe y se siente diferente de la madre, quien se ubica en el lugar de la otredad, lo cual implica que a partir de dicho momento, el niño y la niña deben asumir como suyas sus emociones $y$ afectos, independientemente de su madre $y$ de su valencia - sea esta positiva o negativa-; de manera tal, que los denuestos $y$ ataques fantaseados $y$ proyectados sobre el objeto afectivo que amenazaban con destruirlo, se tienen que asumir como propios, lo que conduce a la clásica posición depresiva kleiniana (Hinshelwood, Robinson y Zárate, 1997).

A su vez, el objeto afectivo se integra $y$ deja de estar escindido como bueno o malo con "o" de exclusión, concibiéndose en la doble posibilidad de gratificar y frustrar. De igual manera, el niño y la niña reconocen para sí la doble posibilidad de amar y odiar, haciéndose cargo de los propios sentimientos. Es así como el sujeto humano se convierte en individuo en virtud de dichos procesos, de manera tal que el no acceder a esta etapa, implica un atrapamiento en las redes simbióticas de la madre que lo condena a la sujeción y a la dependencia.

La asunción de la responsabilidad por los ataques fantaseados y el temor concomitante por el daño infringido, surge de la omnipotencia del pensamiento infantil, ya que por una parte las injurias y agresiones originalmente proyectadas que le amenazaban, se reconocen ahora como propias $y$ por otra parte, se teme la venganza del objeto afectivo, el abandono o su destrucción, ya que lo que se piensa es real y por tanto, tiene consecuencias. Se tiene aquí el origen de la culpa que caracteriza en general, a los procesos depresivos, incluidas las tendencias depresivas que pueden prevalecer como un rasgo constitucional básico tempranamente adquirido.
Los trastornos afectivos pueden desarrollarse a partir de dichos procesos, dando lugar, así como posibilidades a una sobreestimación del objeto afectivo en detrimento de sí mismo, en lo que Freud (1917) denominó melancolía, hasta una sobre estimación propia a despecho del otro - representado por la imago materna- tal y como lo formuló Freud (1914) en el caso de las constituciones narcisistas. De esta manera, el objeto afectivo puede ser idealizado o ser sujeto de odio y desprecio, así como de una relación ambivalente, existiendo también la posibilidad de una vinculación que permita una sana estimación del objeto y de sí mismo.

A partir de estos procesos, los individuos se posicionaran en relación al propio yo $y$ a los otros, con mayor o menor seguridad respecto a su lugar en el mundo circundante $y$ se establecerán tempranamente, determinadas tendencias $\mathrm{u}$ orientaciones vitales básicas que caracterizarán al individuo, tales como: intraversión-extraversión, entre otras posibilidades. Es también el momento de la clásica tarea evolutiva definida por Ericsson (2000) de la "confianza básica versus desconfianza básica".

Desde una perspectiva socio-emocional, otro gran momento del desarrollo del ser humano lo constituye el complejo de Edipo, que ha sido definido como el gran organizador de la personalidad, a partir de su relevancia onto $y$ filogenético. Freud (1921) inspirado en los hallazgos darwinianos, establece que en tiempos de la horda primordial el padre, líder o caudillo, imponía su dominio por la fuerza, hasta que en un momento dado la fratría uniendo sus fuerzas, logró realizar colectivamente lo que individualmente no eran capaces cometiendo parricidio. No obstante, este acto emancipatorio trajo consigo como consecuencia, el que se denegara mutuamente el objeto primordial de deseo que siempre es la madre (Martínez, 2007). Este es el primer contrato social que se suscribe como humanidad (Freud, 1914) y a partir de lo cual, se articulará la superestructura jurídico-política.

El incesto, desde una perspectiva antropológica (Martínez, 2007), es una ley fundante $y$ determinante que establece una prohibición esencial, en primera instancia, entre madre e 
hijo, así como, tío y sobrina en las sociedades matrilineales que caracterizaron el origen de la humanidad, extendiéndose posteriormente a hermanos, primos y en general, al clan que compartía una consanguinidad - real o supuesta - o que al menos reconocían un origen común, esto es el tótem, por lo que en este acto se concebían como parientes. Estos hechos son el fundamento de la exogamia, que como forma de organización y estructura social, se asienta en el tabú del incesto. Este estado de cosas -que se encuentran en la base del surgimiento de la humanidad como tal-, es recreado a nivel ontogenético, a partir del complejo de Edipo, donde los niños y las niñas reviven dicho drama humano en lo individual, perpetuándolo por esta vía a nivel filogenético.

La resolución edípica en Freud implica el rompimiento de la díada madre/hijo e hija y el establecimiento de la triada edípica con la inclusión del padre o su subsidiario. El padre o quien lo representa es quien le dice metafóricamente a la madre y al niño o la niña que: "aquí no se juega de a dos, sino de a tres, yo cuento en esta relación...", estableciéndose de esta manera, la tríada edípica, esto es: madre, padre, hijo o hija. El padre se ubica como el tercero incluido en la relación diádica, como ese otro del deseo de la madre que no es el hijo o la hija. Es ese otro por el cual la madre hace una esquicia en la mirada $y$ vuelve a ver a un tercero que convoca su deseo, desplazando al niño o a la niña como objeto exclusivo de afecto. Es menester aclarar que la función de corte y autoridad asignada a la función paterna ha sido discutida por Benjamin (1996) y otras autoras, quienes indican que es la madre quien impone sus propios límites y obra la separación entre ella y sus hijos e hijas.

Los psicoanalistas que creen que el padre tiene una función de ruptura del vínculo diádico, parten de que este asume un lugar de autoridad, ya que deniega y prohíbe a la madre como objeto afectivo exclusivo, razón por la cual deviene como una otredad que rompe el atrapamiento con el universo simbólico materno. Por otra parte, se han hecho planteamientos en el sentido de que la paternidad es una función que la puede ejercer el padre biológico, el padre de crianza o el compañero de la madre; sin embargo, dicha función y la ruptura del vínculo diádico a ella asociada, puede ser ejercida también por aquello otro que convoca y concita la atención materna, haciendo que la madre ponga su interés en otra cuestión - su trabajo, el estudio o cualquier otra vocación o inclinación- más allá del hijo o la hija.

Solo las madres seguras de sí mismas que confían plenamente en su ejercicio materno, pueden abrirse a la experiencia e introducir a un tercero - incluido otros intereses y vocaciones-y ser capaces de indicarle claro $y$ preciso al hijo $y$ a la hija que tienen un lugar fundamental en su vida, pero que sus anhelos les trascienden.

La resolución edípica en su condición del gran organizador de la subjetividad, es el asiento sobre el cual se estructurará en definitiva el edificio de la personalidad, que tendrá como base $y$ sustento, el periodo vital que va de los tres a los cinco años de vida (Brenner, 1988). En esta etapa se establecerán las principales tendencias, orientaciones y rasgos de carácter, tal y como lo entendió Fromm (1973), las cuales, a partir de dicho momento vital tendrán un carácter más o menos permanente a lo largo de la vida.

En una perspectiva psicodinámica a partir del complejo de Edipo y según sea su resolución a nivel etiopatogénico, se tendrá como posibilidad el surgimiento de las neurosis que clásicamente Freud definió como obsesión, fobia e histeria, aunque originalmente, el propio Freud incluyó las denominadas neurosis de ansiedad. Desde el punto de vista psicoanalítico, estos trastornos comparten una etiología semejante, la cual se caracteriza por el dilema entre el deseo que convoca el objeto afectivo primordial y la denegación que la cultura impone (Freud, 1930). De las tensiones entre las tendencias básicas del individuo y las prescripciones socioculturales, así de cómo dirima el sujeto tales conflictos, dependerá la demostración o no de algunas manifestaciones psicopatólogicas. El caso de la manifestación fóbica de la neurosis estará signada por el temor al castigo, debido a la trasgresión real o imaginada del precepto del incesto. En el caso de las obsesiones, se tiene como resultado la culpa asumida 
por haber accedido de manera real o fantaseada al tabú del incesto. La histeria por su parte se debate en la ambivalencia que suscita la seducción y la prohibición para acceder al objeto primordial del deseo.

No debe obviarse la manera esquemática con que han sido presentadas las formulaciones precedentes, las cuales no agotan las diversas teorizaciones y conclusiones que al respecto se han realizado; sin embargo, para los efectos de este breve trabajo basta en lo expuesto aquí.

En este repaso psicopatológico no puede dejar de mencionarse el espectro de las denominadas perversiones, donde se encuentran dos lecturas disímiles, en cuanto a su concepción $y$ el momento evolutivo en que acaece. Para la Teoría de las Relaciones Objetales, las perversiones son una suerte de fijación pregenital, ergo, corresponden a una sexualidad inmadura, infantil, temprana y generalizada, característica de una detención en el desarrollo psicosexual. Desde el punto de vista psicoanalítico clásico (Freud, 1909,), esta sexualidad se caracteriza por su polimorfía, su adscripción a la díada materno-filial y el no advenimiento del objeto paterno en su condición de ruptura. Clínicamente, no existe una integración del objeto sexual, el cual se caracteriza por su parcialidad.

Para la lectura estructural psicoanalíti$\mathrm{ca}$, el perverso es aquel que conociendo la ley paterna, la desobedece $y$ en este acto, se pone al margen de toda forma jurídica, ya que al no reconocer esta ley primordial, no reconocerá toda legalidad ulterior que se asienta precisamente en el precepto fundacional del tabú del incesto.

En relación a su naturaleza y origen en el desarrollo, ambas explicaciones de las perversiones son disímiles, por cuanto en el primer caso se trata de una sexualidad regresiva, donde el padre no cuenta como objeto, en tanto en el segundo caso se trata del desafío alevoso a un mandato paterno fundamental. Desde el punto de vista evolutivo, las perversiones según las entiende la Teoría de las Relaciones Objetales, ocurren tempranamente - esto es durante la etapa pre-edípica- mientras que para las lecturas estructurales psicoanalíticas, las perversiones son una forma o manera de resolución edípica.

\section{CONCLUSIONES}

A partir de este breve recorrido ha manera de una psicopatología estructural, se puede concluir siempre dentro de esta línea de pensamiento, que el asiento de la psicosis es previo al advenimiento del objeto afectivo, su lugar es el de la indiferenciación y la indiscriminación yoica sin sujeto ni otredad. A su vez, el lugar de los trastornos estructurales, constitucionales $y$ de personalidad, lo constituye la relación diádica de la madre o quien la representa y los hijos e hijas, donde la madre o quien cumpla su función es la que califica la experiencia $y$ define los contornos caracteriales de los individuos. Los denominados trastornos anímicos tendrían por sustrato la etapa de separación-individuación y la culpa concomitante que caracteriza a dicho proceso. Por último, durante la etapa edípica con la introducción del tercero, se brinda el caldo de cultivo para las constituciones y trastornos neuróticos mediados por el deseo que el objeto primordial convoca y la denegación que sobre este, la cultura impone; clásicamente en Freud, las neurosis asumen la modalidad de fobias, obsesiones e histerias (Braunstein, 1993). Las perversiones según hemos dicho, pueden obedecer a una fijación pre-edípica o a un desafío abierto a la ley paterna en correspondencia a determinadas lecturas psicoanalíticas. Desde una perspectiva psicodinámica, el establecimiento de estos momentos evolutivos $y$ sus respectivas formas de resolución, constituyen una importante guía para orientar la acción clínica.

\section{BIBLIOGRAFÍA}

Asociación Americana de Psiquiatría. Diagnostic and Statistical Manual of Mental Disorders (DSm-IV-TR). Barcelona, España: Masson sa, 2002.

Benjamin, Jessica. Los lazos del amor. Psicoanálisis, feminismo y el problema de la dominación. Buenos Aires, Argentina: Paidós, 1996.

Bowlby, John. La pérdida afectiva: tristeza y depresión. Buenos Aires, Argentina: Paidós, 1991. 
Braunstein, Néstor. La clínica psicoanalítica: de Freud a Lacan. San José, Costa Rica: Clínica de Psicología Dinámica, 1993.

Brenner, Charles. Elementos fundamentales de Psicoanálisis. Buenos Aires, Argentina: Editorial Libros Básicos, 1988.

Erickson, Erick. El ciclo vital completado. Barcelona, España: Paidós, 2000.

Freud, Anna. El yo y los mecanismos de defensa. Buenos Aires, Argentina: Paidós, 1954.

Freud, Sigmund (1900). "La interpretación de los sueños". Obras Completas. Tomo II. Madrid, España: Editorial Nueva Biblioteca, 1996.

Freud, Sigmund (1905). "Análisis fragmentario de una histeria". Obras Completas. Tomo I. Madrid, España: Editorial Nueva Biblioteca, 1996.

Freud, Sigmund (1909). "El hombre de las ratas". Obras Completas. Tomo II. Madrid, España: Editorial Nueva Biblioteca, 1996.

Freud, Sigmund (1909). "Análisis de la fobia de un niño de cinco años". Obras Completas. Tomo II. Madrid, España: Editorial Nueva Biblioteca, 1996.

Freud, Sigmund (1909). "Tres ensayos para una teoría sexual". Obras Completas. Tomo II. Madrid, España: Editorial Nueva Biblioteca, 1996.

Freud, Sigmund (1911). "Observaciones psicoanalíticas sobre un caso de paranoia". Obras Completas. Tomo II. Madrid, España: Editorial Nueva Biblioteca, 1996.

Freud, Sigmund (1912). "Totem y tabú". Obras Completas. Tomo II. Madrid, España: Editorial Biblioteca Nueva, 1996.

Freud, Sigmund (1914). "Introducción al narcisismo". Obras Completas. Tomo II. Madrid, España: Editorial Nueva Biblioteca, 1996.

Freud, Sigmund (1915). "Los instintos y sus destinos". Obras Completas. Tomo II. Madrid: Madrid, España: Editorial Biblioteca Nueva, 1996.

Freud, Sigmund (1917). "Duelo y melancolía". Obras Completas Tomo II. Madrid, España: Editorial Nueva Biblioteca, 1996.
Freud, Sigmund (1921). "Psicología de las masas y análisis del yo". Obras Completas. Tomo II. Madrid, España: Editorial Nueva Biblioteca, 1996.

Freud, Sigmund (1927). "El porvenir de una ilusión". Obras Completas. Tomo III. Madrid, España: Editorial Nueva Biblioteca, 1996.

Freud, Sigmund (1930). "El malestar en la cultura". Obras Completas. Tomo III. Madrid, España: Editorial Nueva Biblioteca, 1996.

Freud, Sigmund (1932). "Sobre la sexualidad femenina". Obras Completas. Tomo III. Madrid, España: Editorial Nueva Biblioteca, 1996.

Fromm, Erick y Maccoby, Michael. Sociopsicoanálisis del campesino mexicano: un estudio de la economía y la psicología de una comunidad rural. México DF, México: Fondo de Cultura, 1973.

Hinshelwood, R.; Robinson, S. y Zárate, O. Melanie Klein para principiantes. Buenos Aires, Argentina: Era Naciente, 1997.

Kemberg, Otto. The narcissistic personality disorder and the differential diagnosis of antisocial behavior. Psychiatric Clinics of North America, 1989.

Klein, Melanie. El psicoanálisis de niños. Buenos Aires, Argentina: El Ateneo, 1948.

Liberman, David. Semiótica y psicoanálisis de juego. Buenos Aires, Argentina: Amorrortu Editores, 1984.

Mahler, Margaret. Simbiosis humana: vicisitudes de la individuación. México DF, México: Editorial J. Mortiz, 1972.

Martínez, Manuel. "Psicoanálisis y marxismo una imposibilidad paradigmática". Revista de Ciencias Sociales 115. San José, Costa Rica. Editorial de la Universidad de Costa Rica, 2007: 85-93.

Martínez, Manuel. Constitución de la subjetividad. Reflexiones psicogenéticas. Presentado a la Editorial de la Universidad de Costa Rica, 2013. 
Méndez, Maricela. Manual de los trastornos de personalidad: aspectos clinicos y psicodiagnósticos. San José, Costa Rica: EDNASSs, 2003.

Spitz, Rene. El primer año de vida. México DF, México: Fondo de Cultura Económica, 1977.
Winnicott, D. Realidad y juego. Barcelona, España: Editorial Gedisa, 1982.

Fecha de ingreso: 14/04/2013

Fecha de aprobación: 06/09/2013 